\title{
Isolation, Identification and Antibiotic Susceptibility Patterns of Listeria Monocytogens from Pregnant Women
}

\author{
Brajesh Kumar Jha,' Neetu Adhikari,' R K Sanjana' \\ 'Department of Microbiology, College of Medical Sciences, Bharatpur, Nepal.
}

\section{ABSTRACT}

\section{Introduction}

The present study is made to determine the prevalence rate, isolation, identification and antibiotic susceptibility pattern of L. monocytogens isolated from pregnant women. This research not only guides to the clinician for treatment but also help laboratory worker to monitor susceptibility pattern among the Listeria species.

\section{Methods}

The samples were collected from Gynecology department from January 2017 to December 2019 and processed in the Department of Microbiology at College of Medical Sciences, Bharatpur Nepal. Two hundred thirty four antenatal mothers having age between 17 and 39 years of gestation period between $7^{\text {th }}$ weeks and $36^{\text {th }}$ weeks patient with a flue like sign and symptoms were screened for L. monocytogens. Bacterial isolation identification and antibiotic sensitivity tests were done as per standard microbiological guidelines.

\section{Results}

In this present research, the prevalence of L. monocytogens was found 39/234 (16.7\%), where high rate of Listeria infection was found 21(53.1\%) among the patients of age between 25 and 32 who were mostly from urban area. We found eating meat 38(97.4\%) and fish 39(100.0\%), non-pasteurized simple boiled milk 39(100.0\%) and vegetables like salad eater 32(82.1\%) has highest rate of listeriosis. The results showed most of the listeriosis were occurring in a $3^{\text {rd }}$ trimester $17(43.6 \%)$ compared with the $2^{\text {nd }}$ and $1^{\text {st }}$ trimester. Overall, the antibiotics resistance pattern found low in L. monocytogens. This investigation also showed less resistance in erythromycin 9(23.1\%), ciprofloxacin 8(20.5\%), chloramphenicol $16(41.1 \%)$. The increased resistance pattern among Listeria species were found in penicillin 31(79.5\%), cotrimoxazole 20(51.3\%), amoxicillin 21(55.1\%) and vancomycin $29(74.4 \%)$.

\section{Conclusion}

It is very important to consider listeriosis in a clinical practice now a day. Maternal Listeriosis should be considered in all age group of pregnant women. Regular surveillance of antibiotic susceptibility test is very much important for clinician to select best medicine to treat different cases of listeriosis.

Key words: Listeria monocytogens, pregnant women, drugs resistance.

Correspondence: Brajesh Kumar Jha, Department of Microbiology, College of Medical Sciences, Bharatpur, Nepal. Email: baiju010@gmail.com, Phone: +977-9845087892. 


\section{INTRODUCTION}

Incidence of Listeria monocytogens infection has been significantly increasing over the past few years, mainly affecting pregnant women and immunocompromised patients. The emergence of Zoophilic and Geophilic bacterial infections as the world wide health care problems may be attributed to the extensive use of antibiotics and immunosuppressive agents, as well as an increase in the population of immunocompromised people. ${ }^{1}$ During 1980s a number of large scale outbreaks of Listeriosis occurred, in which contaminated ready to eat foods, uncooked meats, fish, cabbage, milk and cheese were implicated as major source for the disease. In United States listeriosis caused approximately 2500 serious illnesses and 500 deaths annually and $17 \%$ of cases were associated with pregnancy.,3 Infection in pregnant women recognized in third trimester, it may lead to intrauterine fetal infection with a mortality of $30-35 \% .{ }^{4}$ In adult Listeria monocytogens causes meningitis, menigoencephalitis, rhomboencephalitis, brain abscess and spinal cord abscess. It also causes bacteremia and infective endocarditis. Listeriosis represents a significant public health problem and accounts for almost 5-10\% of carriage rate in diseases. ${ }^{5}$

It is a Gram positive bacilli (GPB) belongs to the genus Listeria, occurs worldwide in soil, surface water, vegetation and throughout the food chain. It is recently placed between Lactobacillus and Bacillus, it is closely related to Brocothrix (Seeliger, 1986). ${ }^{6}$ The GPB has six species but only Listeria monocytogens is most likely to be encountered as a human pathogens. In the country like Nepal even it is a potential threat to human beings caused by this ignored gram positive bacilli, there is very less data available on listeriosis among pregnant women in Nepal. Therefore, the aim of present research is to determine the prevalence, isolation, identification and antibiotic susceptibility pattern of L. monocytogens among pregnant women attending antenatal checkup in College of Medical Sciences, Teaching Hospital, Bharatapur, Nepal.

\section{METHODS}

\section{Study area and design}

It is a tertiary care center, hospital based; crosssectional study was conducted from January 2017 to December 2019 at Collage of Medical Sciences, Bharatpur Nepal. Two hundred thirty four antenatal mothers in between $7^{\text {th }}$ weeks to $36^{\text {th }}$ weeks of gestation period patient with a flue like sign and symptoms were screened for L. monocytogens. The subjects selected were having age between 17 and 39 years.

\section{Eligibility criteria}

Pregnant women having influenza like prodorm with chills, coryzal symptoms, myalgia, backache, headache, vomiting/ diarrhea and urinary frequency was included in the present research.

\section{Sample size and sampling technique}

A total of 234 antenatal mothers who were clinically diagnosed as influenza like illness, selectively recruited from January 2017 to December 2019 for the present study.

\section{Dependent variables}

Prevalence of L. monocytogens in antenatal mother and its antimicrobial susceptibility test. 


\section{Independent variables}

Socio-demographic variables.

\section{Sample collection}

The clinical specimen and demographic data were collected using ethically approved questionnaire. After taking informed written consent from patients or patient attendant guardian, $5 \mathrm{ml}$ of blood sample was aseptically collected and transferred into a sterile Tryptose soy broth in a screw caped test tube, transported immediately to the laboratory without delay within 1 hour. It was incubated $37^{\circ} \mathrm{C}$ for overnight and turbidity in the broth was observed in the next day.

\section{Culture and identification}

The turbid Tryptose soy broth were further subcultured on Palkam agar media and incubated at $37^{\circ} \mathrm{C}$ for $24 \mathrm{hr}$. the specific selective growth of green shiny colonies having diffuse black shadow on Palkam agar were taken as a Listeria species. If there was no growth found on palkam agar, those plates were further incubated for another 24 hours. Those Listeria spp. were further identified using conventional methods, viz gram stain, catalase test, motility test, hemolysis on blood agar, CAMP test, IMViC tests and sugar fermentation tests (xylose, mannito, rhamnose and methyl D-mannopyranoside). ${ }^{7}$

\section{Antibiotic susceptibility tests}

Antibiotic sensitivity tests were done as per standard microbiological guide line by KirbyBauer disc diffusion method. ${ }^{8}$ the Listeria monocytogens bacterial isolates inoculum suspension was prepared in a sterile saline and turbidity were compared using 0.5 McFarland standard solution. The Mueller Hinton agar
(MHA) plates were inoculated with bacterial saline suspension dipped sterile cotton swab uniformly. Those inoculated MHA plates were incubated for 10 minutes for drying, than antibiotic discs were further placed on it. The antibiotic discs used were Amoxycillin (30mcg/disc), Co-trimoxazole (25mcg/disc), Ciprofloxacin (5mcg/disc), Chloramphenicol (30mcg/disc), Erythromycin (15mcg/disc), Penicillin (10units/disc), Clindamycin (2mcg/ disc) and Vancomycin (30mcg/disc), HiMedia, India.

\section{Quality assurance and data analysis}

The standard microbiological procedure were followed using reference strain of $L$. monocytogens (ATCC 7644) was used as quality control strain which were obtained from PGI Chandigarh, India. All the data were analyzed using SPSS version 20. Comparisons of species distribution and antifungal susceptibility test were determined by the chi-square test for categorical variables and the Wilcoxon rank sum test for ordinal variables. Binary logistic regression was used to show the association of each variable with the dependent variable. A p-value $<0.05$ indicated statistical significance between any compared mean groups.

\section{RESULTS}

In the present study, A total number of 234 samples of suspected cases of Listeriosis were processed where the prevalence of L. monocytogens was found 39/234 (16.7\%). The high rate of Listeria infection was found $21(53.1 \%)$ among the patients of age between 25 and 32 who were mostly from urban area $26(66.7 \%)$. Patients with mud house $26(66.7 \%)$, farmer 20(51.3\%), house wife $12(30.8 \%)$ and cat as pet animals $24(53.8 \%)$ in their house 
were using pipe water $14(35.9 \%)$ has more incidence of listeriosis compared to other group of people in the community as shown in Table -1 .

Table 1. Association of socio-demographic characters in a $L$. monocytogens isolation from pregnant women visited to the hospital with clinical features of listeriosis.

\begin{tabular}{|c|c|c|c|c|}
\hline \multirow[t]{2}{*}{ Variable } & \multirow[t]{2}{*}{ Number } & \multirow[t]{2}{*}{ Percentage } & \multicolumn{2}{|c|}{ L. monocyłogens } \\
\hline & & & Positive & Negative \\
\hline \multicolumn{5}{|l|}{ Age } \\
\hline $17-24$ & 46 & 19.7 & 11 & 35 \\
\hline $25-32$ & 161 & 68.8 & 21 & 140 \\
\hline 33-39 & 27 & 11.5 & 7 & 20 \\
\hline \multicolumn{5}{|l|}{ Residence } \\
\hline Urban & 45 & 19.2 & 26 & 19 \\
\hline Rural & 189 & 80.7 & 13 & 176 \\
\hline \multicolumn{5}{|c|}{ Knowledge of Hygiene } \\
\hline Yes & 31 & 13.3 & 5 & 26 \\
\hline No & 203 & 86.7 & 34 & 169 \\
\hline \multicolumn{5}{|l|}{ Occupation } \\
\hline House wife & 86 & 36.7 & 12 & 74 \\
\hline Farmer & 85 & 36.3 & 20 & 65 \\
\hline Office worker & 63 & 29.9 & 7 & 56 \\
\hline
\end{tabular}

Source of water use

\begin{tabular}{|c|c|c|c|c|}
\hline Piped & 98 & 41.8 & 14 & 84 \\
\hline Bore well & 71 & 30.4 & 10 & 61 \\
\hline Supply water & 65 & 27.8 & 5 & 60 \\
\hline
\end{tabular}

Keeping pets

\begin{tabular}{|c|c|c|c|c|}
\hline Cat & 42 & 17.9 & 21 & 21 \\
\hline Dog & 165 & 70.5 & 15 & 150 \\
\hline Dog and Cat & 27 & 11.5 & 3 & 24 \\
\hline
\end{tabular}

Type of living room

\begin{tabular}{|c|c|c|c|c|}
\hline Mud house & 195 & 83.3 & 26 & 169 \\
\hline Cement house & 39 & 16.7 & 13 & 26 \\
\hline
\end{tabular}

We found eating meat 38(97.4\%) and fish $39(100.0 \%)$, non-pasteurized simple boiled milk 39(100.0\%) and vegetables like salad eater $32(82.1 \%)$ has highest rate of listeriosis. The results showed most of the listeriosis were occurring in a $3^{\text {rd }}$ trimester $17(43.6 \%)$ compared with the $2^{\text {nd }}$ and $1^{\text {st }}$ trimester (Table-2).

Table 2. Association of risk factors for Listeriosis from the pregnant women attending antenatal care clinic in CMS-TH, Bharatpur, Nepal.

\begin{tabular}{|l|l|l|l|l|}
\hline Variable & Number & Percentage & L. monocytogens \\
\cline { 3 - 4 } & & Positive & Negative \\
\hline
\end{tabular}

Eating habit

\begin{tabular}{|c|c|c|c|c|}
\hline Meat \\
\hline Yes & 222 & 84.9 & 38 & 184 \\
\hline No & 12 & 5.1 & 1 & 11 \\
\hline
\end{tabular}

Fish

\begin{tabular}{|c|c|c|c|c|}
\hline Yes & 224 & 95.7 & 39 & 185 \\
\hline No & 10 & 4.3 & 0 & 10 \\
\hline \multicolumn{5}{|l|}{ Boiled milk } \\
\hline Yes & 226 & 96.6 & 39 & 187 \\
\hline No & 8 & 3.4 & 0 & 8 \\
\hline
\end{tabular}

Uncooked vegetables (Salad)

\begin{tabular}{|c|c|c|c|c|}
\hline Yes & 210 & 89.7 & 32 & 178 \\
\hline No & 24 & 10.3 & 7 & 17 \\
\hline
\end{tabular}

Gestational period

\begin{tabular}{|c|c|c|c|c|}
\hline $1^{\text {st }}$ trimester & 154 & 65.8 & 8 & 146 \\
\hline $2^{\text {nd }}$ trimester & 52 & 22.2 & 14 & 38 \\
\hline $3^{\text {rd }}$ trimester & 28 & 12.0 & 17 & 11 \\
\hline
\end{tabular}

Overall, Table-3 and Figure-1 shows the antibiotics resistance pattern was low in $L$. monocytogens. This investigation also reveled less resistance in erythromycin 9(23.1\%), ciprofloxacin $8(20.5 \%)$, chloramphenicol $16(41.1 \%)$. The increased resistance pattern among Listeria species were found in drugs like penicillin 31(79.5\%) cotrimoxazole 20(51.3\%), amoxicillin $21(53.1 \%)$ and vancomycin $29(74.4 \%)$. 


\begin{tabular}{|c|c|c|c|c|}
\hline \multirow{2}{*}{ Antibiotics } & \multicolumn{2}{|c|}{ Sensitive } & \multicolumn{2}{|c|}{ Resistance } \\
\hline & Number & Percentage & Number & Percentage \\
\hline Penicillin & 8 & 20.5 & 31 & 79.5 \\
\hline Co-trimoxazole & 19 & 48.7 & 20 & 51.3 \\
\hline Amoxycillin & 18 & 46.2 & 21 & 53.8 \\
\hline Erythromycin & 30 & 76.9 & 9 & 32.1 \\
\hline Vancomycin & 10 & 25.6 & 29 & 74.4 \\
\hline Clindamycin & 25 & 64.1 & 14 & 35.9 \\
\hline Ciprofloxacin & 31 & 79.5 & 8 & 20.5 \\
\hline Chloramphenicol & 23 & 58.9 & 16 & 41.1 \\
\hline
\end{tabular}

$\mathrm{Hi}$

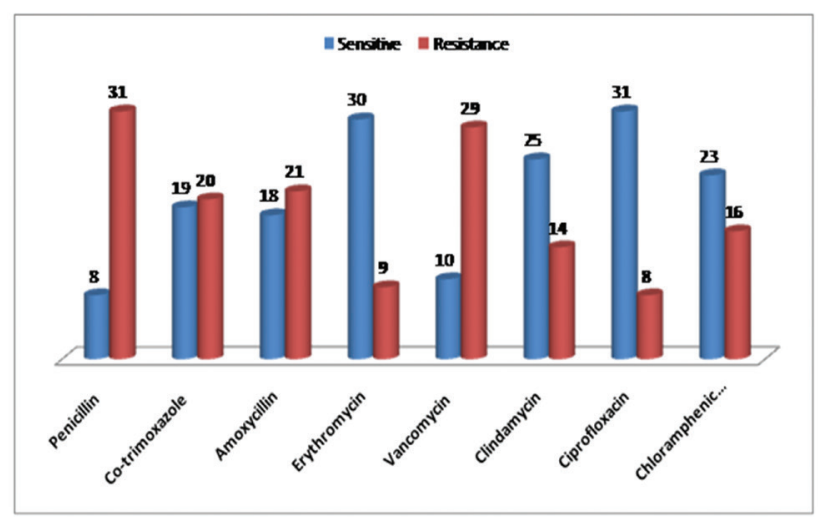

Figure 1. Shows the susceptibility patterns among isolated $L$. monocytogens.

\section{DISCUSSION}

In this present research, the prevalence of $L$. monocytogens was found 39/234 (16.7\%) clinically suspected listeriosis among pregnant women. This prevalence rate is higher compared to previous studies, where rate of prevalence was $0.1 \%$ reported. This is due to selectively taken clinically suspected cases of Listeria infection among patients. Listeriosis is a disease that can cause mild asymptomatic infection to devastating fetal problems in pregnant women like, preterm delivery, stillbirth, and fetal death. In few cases, it may complicate to serious neonatal morbidity viz septicemia, pneumonia, meningitis and encephalitis.

The percentage of Listeria monocytogens in our study was $16.7 \%$ compared to other bacterial infections which accounted for $83.3 \%$. Further, pathogenicity study was essential to prove pathogenic role of isolated Listeria species. Different study has proven the close association of Listeriosis and immunocompromised condition of patients. , $^{910}$

In our study, pregnant women with age between 25 and 32 had high rate of Listeria infection $21(53.1 \%)$ and most of the patients were from urban area $26(66.7 \%)$. These results were in accordance with the publisher's report, who also reported rural area with all age group patients has more chances to get Listeria infection compared to urban area. ${ }^{11}$ As we know hygiene has inverse relation with the bacterial infection, therefore similar pattern of result were obtained in the present scenario, where we found unhygienic patients with more listeriosis.

In the present research, patients with mud house $26(66.7 \%)$, farmer 20(51.3\%), house wife $12(30.8 \%)$ and cat as pet animas $21(53.8 \%)$ in their house were using pipe water $14(35.9 \%)$ has more incidence of listeriosis compared to other 
group of people in the community. Similar type of data were also obtained in another research done by (Linke et al, 2018). ${ }^{12}$ It is well known that Listeria species is a ubiquitous bacteria therefore, it has high chances to cause infection in low immunity host like pregnant women. ${ }^{13}$

Maternal listeriosis is usually mild but it is very sever for neonates. Hence, the current research emphasizes the need to screen hygienic condition and gestational period of infection which also gives us clue for association of bacteria with food habits among patients. We found eating meat $38(97.4 \%)$ and fish $39(100.0 \%)$, non-pasteurized simple boiled milk 39(100.0\%) and vegetables like salad eater $32(82.1 \%)$ has highest rate of listeriosis. Mostly similar types of results were also seen in different research, where farmer, domestic animals in their house and eating habits showed high rate of listeriosis. ${ }^{14}$

There are some research which shows time period of pregnancy and its close association of Listeria infection ${ }^{15}$ the similar pattern of research result was also found in our reaearch regarding gastesnal period. The results showed most of the listeriosis were occurring in a $3^{\text {rd }}$ trimester $17(43.6 \%)$ compared with the $2^{\text {nd }}$ and $1^{\text {st }}$ trimester. It is due to sudden change in hormonal status of a pregnant woman. These types of reports were also found from the Ethopia and Uganda. ${ }^{16}$ The developing countries has similar pattern of infectivity rate where there is less practice of hygiene.

Overall, the antibiotics resistance pattern was currently low in L. monocytogens. This investigation also showed quite comparable less resistance in erythromycin 9(23.1\%), ciprofloxacin 8(20.5\%), chloramphenicol $16(41.1 \%)$. The increased resistance pattern among Listeria species were found in penicillin $31(79.5 \%)$ cotrimoxazole $20(51.3 \%)$, amoxicillin $21(53.8 \%)$ and vancomycin $29(74.4 \%)$. This type of resistance pattern shows red alarm and also makes us more attentive towards the drug susceptibility pattern among Listeria species. This may be due to acquaring resistance or plasmid mediated resistance among the Listeria species as reported by (Emmanuelle and Patrice, 1999). ${ }^{17}$ It is very much confirmatory that Listeria might have acquired drug resistance gene from different sources by different modes.

\section{Limitations}

All Listeria species isolated should be characterized by molecular technique. Virulence factor and Pathogenicity should be continuous monitored by performing research. Routine antibiotics susceptibility test must be performed to see the resistance among the isolates.

\section{CONCLUSIONS}

In the current scenario of mostly ignored gram positive rods Listeria infection is increasing day by day. It is very important to consider listeriosis in a clinical practice now a day. Maternal Listeriosis should be considered in all age group of pregnant women due to diligence must be followed in a lab. It is mostly a food borne disease therefore; a government guideline must be made to ensure food safety.

\section{ACKNOWLEDGEMENTS}

The Author would like to thank College of Medical Sciences, Bharatpur, Nepal for allowing me to do this research work in the laboratory, Ganesh Singh for helping in sample processing and my wife Mamta Kumari Jha for typing manuscript. 


\section{REFERENCES}

1. Ashley A, DeNegre, Martial L et al. Emergence of antibiotic resistance in immunocompromised host populations: A case study of emerging antibiotic resistant tuberculosis in AIDS patients. Plus one. 2019:1-13.

2. Conly JM, Johnston BL. Listeria: A persistent food-borne pathogen. Can J Infect Dis Med Microbiol 2008;19 (5): 327-328.

3. Catherine WD. Listeria monocytogenes: A Continuing Challenge. Pub med. 2001; 59(6):183-94.

4. Allerberger F Wagner M. Listeriosis: a resurgent food borne infection. Clinic Microbiol and Infect. 2010; 16: 16-23.

5. Kuldeep D, Kumaragurubaran K, Ruchi $\mathrm{T}$ et al. Listeriosis in animals, its public health significance (food-borne zoonosis) and advances in diagnosis and control: a comprehensive review. 2015; 35 (4):211-235.

6. Maurice B, Lai SC, Ahmad ST. Prevalence, Genetic Heterogeneity, and Antibiotic Resistance Profile of Listeria spp. and Listeria monocytogenes at Farm Level: A Highlight of ERIC- and BOX-PCR to Reveal Genetic Diversity Lesley . Hindawi. 2018: 1-13.

7. DeeptiNN,SavaliaCV,KalyaniIH.Isolation, identification, and characterization of Listeria spp. from various animal origin foods. Published online. 2015; 8(6): 695-701.

8. Jan H. Kirby-Bauer Disk Diffusion Susceptibility Test Protocol. ASM 2009; 1-23.

9. Ruchir C, Caitlin K, Syed Q, et al.
Uncommon manifestations of Listeria monocytogenes infection. BMC Infect Dis. 2014; 14: 641.

10. Okutani A. Nationwide survey of human Listeria monocytogenes infection in Japan. Epidemiol Infect. 2004;132: 769-72.

11. Cresence V, Dharsana $K$, Lekshmi M, et al. Listeria review of epidemiology and pathogenesis. J Microbiol Immunol Infect. 2007; 40: 4-13.

12. Kristina Linke et al. Reservoirs of Listeria species in Three Environmental Ecosystems, a Alexander. Applied and Environ Microbiol. 2014; 80 (18): p. 5583-92.

13. Lavious TM, Anthony I. Listeria monocytogenes Virulence, Antimicrobial Resistance and Environmental Persistence: A Review. MDPI 2020; 1-12.

14. Kuldeep Dhama. Listeriosis in animals, its public health significance (food-borne zoonosis) and advances in diagnosis and control: a comprehensive review. Veterinary Quarterly. 2015; 35 (4): 211-35.

15. Hila E. Pregnancy-Associated Listeriosis: Clinical Characteristics and Geospatial Analysis of a 10-Year Period in Israel. CID. 2014; 59: 953-961.

16. Simon G, Tesfu K, Haile A et al. Isolation and characterization of Listeria monocytogenes and other Listeria species in foods of animal origin in Addis Ababa, Ethiopia. J Infect Public Health. 2011; 4(1):22-9.

17. Emmanuelle C, Patrice C. Antibiotic Resistance in Listeria spp. Antimicrob Agent and Chemothera. 1999; 43(9): p. 2103-8.

Citation: Jha BK, Adhikari N, Sanjana RK, Isolation, Identification and Antibiotic Susceptibility Patterns of Listeria Monocytogens from Pregnant Women. JCMS Nepal. 2021 ; 17(3); 227-33. 